\title{
HABITOS ALIMENTARIOS DE PLEURODEMA THAUL (ANURA, LEPTODACTYLIDAE), EN CONCEPCION, CHILE
}

\author{
FEEDING HABITS OF PLEURODEMA THAUL (ANURA, \\ LEPTODACTYLIDAE), IN CONCEPCION, CHILE
}

\author{
Helen Díaz-Páez \& Juan Carlos Ortiz \\ Departamento de Zoología, Facultad de Ciencias Naturales y Oceanográficas, Universidad de Concepción, \\ Casilla 160-C, Concepción, Chile
}

\begin{abstract}
RESUMEN
Se analizó la dieta de una población del sapito de cuatro ojos (Pleurodema thaul) de la localidad de Hualpén (Talcahuano, Chile). El estudio se realiza durante los períodos reproductivos (junio-diciembre) que comprenden los años 2000 y 2001. Estos anuros destinan una menor importancia al consumo de presas durante la época reproductiva, por lo que presentan una baja frecuencia de ocurrencia $(50,63 \%)$. La dieta de $P$. thaul está compuesta fundamentalmente de artrópodos $($ IRI $=0,92)$, con un consumo de menor importancia de vegetales (IRI $=0,035)$. Se determinó una alta diversidad de ítemes de presa (37 taxa). Los ítemes alimentarios dípteros y arácnidos son los más comunes. No se detectan relaciones entre el tamaño de las presas y el tamaño de los anuros.
\end{abstract}

Palabras Claves: Dieta, Anfibia, Leptodactylidae, Chile.

\section{ABSTRACT}

We examined the diet for four eyes toad (Pleurodema thaul) in Hualpén (Talcahuano, Chile). The study was carried out during the breeding season (June - December) in 2000 and 2001. These anurans consume fewer preys during the breeding season, and they present a low occurrence frequency $(50.63 \%)$. The results show the $P$. thaul diet include for arthropods (IRI $=0.92$ ), with a lower consumption of vegetables (IRI $=0.035)$. We determined a high diversity of prey, with 37 taxa of invertebrates. The alimentary items dipteral and arachnids are the most common. No relationships occurred between the size of the preys and the size of the anurans.

KeYwords: Diet, Amphibia, Leptodactylidae, Chile.

\section{INTRODUCCION}

Los hábitos alimentarios proporcionan información acerca de la historia de vida y patrones conductuales de los anuros (Wells 1978; Murphy 1992). Los estudios han determinado que los anuros consumen gran cantidad de invertebrados en condiciones naturales (Whitaker et al. 1977) y el tamaño del cuerpo a menudo influye en la selección de las presas (Boice \& Williams 1971).

Estos antecedentes han llevado a determinar una influencia del hábitat sobre las poblaciones de anfibios, donde las modificaciones de éste afectan la dieta y la estructura de las poblaciones (Anderson \& Mathis 1999; Anderson et al. 1999; Beebee 1996; Krzysik 1979; Toft 1980, 1981). La dieta se transforma entonces en un importante componente de la historia natural de las especies, y sugiere consecuen- 
cias ecológicas en la vida de estas en los diferentes hábitat (Anderson \& Mathis 1999).

En Chile, la información acerca de los hábitos alimentarios de anfibios es escasa y deficiente, donde la mayor parte sólo menciona datos puntuales y de dietas en laboratorio (Cei 1962, 1980; PincheiraDonoso 2002a, 2002b; Valencia et al. 1982). Núñez et al. (1982) muestran diferencias en las dietas de dos poblaciones de B. spinulosus, las que se relacionan con la altitud.

La especie Pleurodema thaul ("sapo de cuatro ojos") es uno de los anuros más comunes de los humedales chilenos, con una distribución que abarca desde la II a XII regiones (Formas 1995). Sin embargo, para $P$. thaul sólo se conoce la dieta en condiciones de cautiverio a base de lombrices de tierra (Cei 1962).

Los objetivos del presente trabajo son: determinar el tipo de presa, su cantidad e importancia relativa en la dieta de los adultos de $P$. thaul; y la relación entre los tamaños de presa y el tamaño del depredador.

\section{MATERIALESY METODOS}

Composición de la dieta. Se realizaron visitas periódicas desde junio de 2000 a diciembre de 2001, a la estación de Biología Terrestre de la Universidad de Concepción, ubicada en el Parque Pedro del Río Zañartu, en Hualpén, Concepción ( $36^{\circ} 47^{\prime} \mathrm{S}, 7^{\circ} 02^{\prime} \mathrm{O}$; 10 m.s.n.m). Los ejemplares fueron capturados manualmente entre las 22:00 y 03:00 h. Cada animal fue pesado $(0,1 \mathrm{~g}$ de precisión) y medido la longitud hocico-cloaca (LHC) con un Vernier de $0,5 \mathrm{~mm}$ de precisión. Los contenidos estomacales se obtuvieron mediante la técnica de "Stomach Flushing" (Legler \& Sullivan 1979; Leclerc \& Courtois 1993). En el laboratorio se analizaron las muestras bajo una lupa estereoscópica Olympus SZ40. Los ítemes de invertebrados fueron determinados hasta el nivel de familia (Peña 1988; Artigas 1994a, 1994b), mientras que los vegetales sólo fueron considerados como restos sin determinar.

Para cada ítem se registró el número total por individuo, y fueron medidos el largo, ancho y grosor promedio utilizando un ocular micrométrico. El volumen de cada presa se estimó al considerar sus formas como cuerpos geométricos sólidos, cilíndricos o rectangulares (Jones 1982; Maiorana 1978;
Petranka 1984). Con estos datos se calculó el porcentaje agregado de volúmenes y el número de ítemes de consumo para cada especie (Litvaitis et al. 1994).

Relación entre el tamaño presa-depredador. Para estimar la relación entre el tamaño de presa y el tamaño del depredador se realizó una regresión entre los volúmenes de los mayores y menores ítemes de presa versus el tamaño del depredador (LHC).

Composición de la dieta. Se determinó un índice de importancia relativa (IRI; Powell et al. 1990) para cada taxón de invertebrados en los ítemes de consumo por año, cuya formula es la siguiente:

$$
I R I=(N+W+F) / 3
$$

donde $\mathrm{N}$ es el porcentaje numérico agregado, $\mathrm{W}$ es el volumen porcentual agregado y $\mathrm{F}$ la frecuencia de ocurrencia. Este índice permite comparar la importancia relativa que cada ítem representa en la dieta total de la especie depredadora.

Los valores de IRI fueron utilizados para estimar la relación entre el tamaño de presa y el tamaño del depredador a través de la regresión de los volúmenes de los mayores y menores ítemes de presa versus el tamaño de los anuros (LHC).

En forma adicional, se utilizó el volumen como un índice del tamaño de presa. A partir de esto se examinó la relación taxonómica específica presadepredador, a través de la regresión de los volúmenes de los ítemes alimentarios más comunes dentro de los tres órdenes de artrópodos más abundantes (díptera, arácnida, y anfípoda), versus el tamaño corporal de los anuros, mientras que la frecuencia de ítemes alimentarios y el número de estómagos que contienen alimento son comparados entre años usando el test $\mathrm{G}(\alpha=0,05)$ (Anderson et al. 1999).

Se comparó la dieta entre años, calculando la amplitud de nicho trófico (DB) durante los años 2000 y 2001 (Pianka 1974). La amplitud de nicho trófico se basó en el índice de diversidad de Simpson (1949):

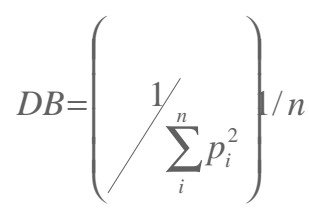

donde pi es la proporción del recurso i-esimo (volumen o número). Cuando se divide por el número de categorías usadas (n), el índice varía entre 0 (indica un bajo rango dietario) y 1 (indica un amplio rango dietario). 
Dieta de Pleurodema thaul. Díaz-PÁez, H. \& Juan C. Ortiz

TABLA I: Datos dietarios para $P$. thaul: En negrita valor de importancia; en paréntesis, número total de presas, volumen total de presas $(\mathrm{ml})$, ocurrencia de cada tipo de presa.

TABLE I: Dietary data for $P$. thaul. Importance value (in Boldface); in parenthesis, total number of preys, volume of preys $(\mathrm{ml})$, frequency of prey type.

\begin{tabular}{|c|c|c|}
\hline Item de presa & $\begin{array}{l}\text { P. thaul } 2000 \\
\quad(\mathrm{n}=47\end{array}$ & $\begin{array}{l}P . \text { thaul } 2001 \\
(\mathrm{n}=34)\end{array}$ \\
\hline \multicolumn{3}{|l|}{ INSECTA } \\
\hline COLEOPTERA & & \\
\hline Anthicidae & $\begin{array}{c}\mathbf{0 , 0 3 6} \\
(10 / 0,0079 / 7)\end{array}$ & - \\
\hline Carabidae & $\begin{array}{c}\mathbf{0 , 0 2 0} \\
(3 / 0,0211 / 3)\end{array}$ & $\begin{array}{c}\mathbf{0 , 0 0 8} \\
(1 / 0,0084 / 1)\end{array}$ \\
\hline Carabidae (larva) & - & $\begin{array}{c}\mathbf{0 , 0 6 5} \\
(7 / 0,1483 / 5)\end{array}$ \\
\hline Elatheridae (larva) & $\begin{array}{c}\mathbf{0 , 0 0 5} \\
(1 / 0,0019 / 1)\end{array}$ & - \\
\hline Helodidae & $\begin{array}{c}\mathbf{0 , 0 0 6} \\
(1 / 0,0086 / 1)\end{array}$ & - \\
\hline Hidrophylidae & $\begin{array}{c}\mathbf{0 , 0 1 3} \\
(1 / 0,0457 / 1)\end{array}$ & - \\
\hline Latrirididae & - & $\begin{array}{c}\mathbf{0 , 0 0 9} \\
(1 / 0,0098 / 1)\end{array}$ \\
\hline Staphilinidae & - & $\begin{array}{c}\mathbf{0 , 0 1 6} \\
(4 / 0,0023 / 2)\end{array}$ \\
\hline $\begin{array}{l}\text { DIPTERA } \\
\text { Drosophilidae }\end{array}$ & $\begin{array}{c}\mathbf{0 , 0 4 8} \\
(11 / 0,0155 / 8)\end{array}$ & $\begin{array}{c}\mathbf{0 , 0 8 0} \\
(15 / 0,0214 / 11)\end{array}$ \\
\hline Drosophilidae (pupa) & $\begin{array}{c}\mathbf{0 , 0 0 6} \\
(1 / 0,0007 / 1)\end{array}$ & - \\
\hline Muscidae & $\begin{array}{c}\mathbf{0 , 1 3 9} \\
(23 / 0,5650 / 9)\end{array}$ & $\begin{array}{c}\mathbf{0 , 0 4 9} \\
(8 / 0,0381 / 6)\end{array}$ \\
\hline Tabanidae (larva) & $\begin{array}{c}\mathbf{0 , 0 1 1} \\
(2 / 0,0009 / 2)\end{array}$ & - \\
\hline Tipulidae & $\begin{array}{c}\mathbf{0 , 2 0 0} \\
(55 / 0,2650 / 22)\end{array}$ & $\begin{array}{c}\mathbf{0 , 2 4 9} \\
(66 / 0,3556 / 22)\end{array}$ \\
\hline Tipulidae (larva) & - & $\begin{array}{c}\mathbf{0 , 0 2 2} \\
(3 / 0,0102 / 3)\end{array}$ \\
\hline HEMIPTERA & & - \\
\hline Saldidae & $\begin{array}{c}\mathbf{0 , 0 0 5} \\
(1 / 0,0002 / 1)\end{array}$ & \\
\hline $\begin{array}{l}\text { HYMENOPTERA } \\
\text { Formicidae }\end{array}$ & $\begin{array}{c}\mathbf{0 , 0 2 4} \\
(7 / 0,0022 / 4)\end{array}$ & - \\
\hline Sphesidae & - & $\begin{array}{c}\mathbf{0 , 0 1 6} \\
(2 / 0,0122 / 2)\end{array}$ \\
\hline Braconidae & $\begin{array}{c}\mathbf{0 , 0 0 5} \\
(1 / 0,0004 / 1)\end{array}$ & - \\
\hline $\begin{array}{l}\text { HOMOPTERA } \\
\text { Aphididae }\end{array}$ & $\begin{array}{ll} & \mathbf{0 , 0 3 2} \\
(6 / 0,0017 / 6)\end{array}$ & - \\
\hline Delphacidae & $\begin{array}{l}\mathbf{0 , 0 2 4} \\
(4 / 0,0118 / 4)\end{array}$ & - \\
\hline $\begin{array}{l}\text { LEPIDOPTERA } \\
\text { Geometridae (larva) }\end{array}$ & $\begin{array}{c}\mathbf{0 , 0 3 7} \\
(3 / 0,0986 / 3)\end{array}$ & $\begin{array}{c}\mathbf{0 , 0 5 7} \\
(4 / 0,1791 / 3)\end{array}$ \\
\hline Geometridae (pupa) & $\begin{array}{c}\mathbf{0 , 0 2 3} \\
(10 / 0,0078 / 3)\end{array}$ & $\begin{array}{c}\mathbf{0 , 0 3 3} \\
(11 / 0,0083 / 4)\end{array}$ \\
\hline $\begin{array}{l}\text { NEUROPTERA } \\
\text { Crysopidae }\end{array}$ & 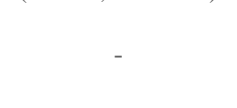 & $\begin{array}{c}\mathbf{0 , 0 1 0} \\
(2 / 0,0139 / 1)\end{array}$ \\
\hline
\end{tabular}


Gayana 67(1), 2003

Continuación Tabla I

\begin{tabular}{|c|c|c|}
\hline Item de Presa & $\begin{array}{l}\text { P. thaul } 2000 \\
(\mathrm{n}=47)\end{array}$ & $\begin{array}{l}\text { P. thaul } 2001 \\
(\mathrm{n}=34)\end{array}$ \\
\hline ORTHOPTERA (restos) & $\begin{array}{c}\mathbf{0 , 0 0 6} \\
(1 / 0,0010 / 1)\end{array}$ & $\begin{array}{c}\mathbf{0 , 0 0 7} \\
(1 / 0,0008 / 1)\end{array}$ \\
\hline \multicolumn{3}{|l|}{$\begin{array}{l}\text { CHELICERATA } \\
\text { AR' CHNIDA }^{\prime} \text { CHN }\end{array}$} \\
\hline Anyphanidae & $\begin{array}{c}\mathbf{0 , 0 6 6} \\
(12 / 0,1442 / 8)\end{array}$ & $\begin{array}{c}\mathbf{0 , 0 2 6} \\
(3 / 0,0254 / 3)\end{array}$ \\
\hline Araneae & $\begin{array}{c}\mathbf{0 , 0 4 0} \\
(9 / 0,0312 / 7)\end{array}$ & $\begin{array}{c}\mathbf{0 , 0 4 4} \\
(8 / 0,0338 / 5)\end{array}$ \\
\hline Clubionidae & $\begin{array}{c}\mathbf{0 , 0 2 0} \\
(5 / 0,0045 / 4)\end{array}$ & $\begin{array}{c}\mathbf{0 , 0 0 7} \\
(1 / 0,0011 / 1)\end{array}$ \\
\hline \multicolumn{3}{|l|}{ ACARI } \\
\hline Hydrozetoidea & $\begin{array}{c}\mathbf{0 , 0 1 0} \\
(3 / 0,0001 / 2)\end{array}$ & $\begin{array}{c}\mathbf{0 , 0 1 6} \\
(4 / 0,002 / 2)\end{array}$ \\
\hline Liacaroidea & $\begin{array}{c}\mathbf{0 , 0 3 2} \\
(12 / 0,0002 / 6)\end{array}$ & $\begin{array}{c}\mathbf{0 , 0 1 6} \\
(4 / 0,0001 / 2)\end{array}$ \\
\hline Trombidiidae & $\begin{array}{c}\mathbf{0 , 0 0 6} \\
(2 / 0,0031 / 1)\end{array}$ & - \\
\hline Oppioidea & $\begin{array}{c}\mathbf{0 , 0 0 5} \\
(1 / 0,0001 / 1)\end{array}$ & - \\
\hline Digamasellidae & $\begin{array}{c}\mathbf{0 , 0 0 5} \\
(1 / 0,0001 / 1)\end{array}$ & - \\
\hline CRUSTACEA & & \\
\hline O. Amphipoda & $\begin{array}{c}\mathbf{0 , 0 3 0} \\
(6 / 0,0160 / 5)\end{array}$ & $\begin{array}{c}\mathbf{0 , 0 8 4} \\
(26 / 0,0738 / 8)\end{array}$ \\
\hline O. Isopoda & $\mathbf{0 , 0 3 3}$ & $\mathbf{0 , 0 3 0}$ \\
\hline Oniscidae & $(17 / 0,0092 / 5)$ & $(6 / 0,0040 / 4)$ \\
\hline Huevos & $\begin{array}{c}\mathbf{0 , 0 3 5} \\
(20 / 0,0002 / 4)\end{array}$ & $\begin{array}{c}\mathbf{0 , 0 6 7} \\
(79 / 0,0002 / 2)\end{array}$ \\
\hline Vegetales & $\begin{array}{c}\mathbf{0 , 0 3 6} \\
(11 / 0,020 / 5)\end{array}$ & $\begin{array}{c}\mathbf{0 , 0 3 4} \\
(6 / 0,0005 / 5)\end{array}$ \\
\hline
\end{tabular}

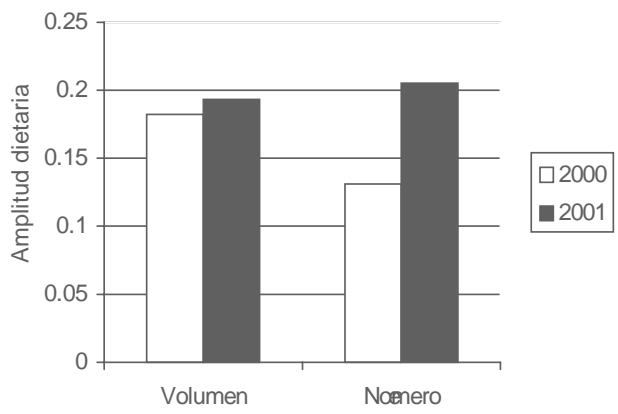

Figura. 1: Amplitud dietaria, DB (Pianka 1974). Basado en los ítem de presa consumidos por Pleurodema thaul en Hualpén durante los años 2000 y 2001.

Figure. 1: Diet breadth, DB (Pianka 1974). Based in the prey ítems consumed for Pleurodema thaul in Hualpén during 2000 and 2001.

\section{RESULTADOS}

\section{COMPOSICIÓN DE LA DIETA}

Se recolectaron 160 ejemplares de $P$. thaul, de los cuales 81 presentaban contenido estomacal. La dieta comprendió un total de 502 presas repartidas en 37 taxa (Tabla I). La frecuencia de los estómagos que contienen presas no presentaron diferencias entre años $(\mathrm{G}=8.98 ; 1 \mathrm{gl} ; \mathrm{P}>0.05)$. El análisis estomacal determinó la presencia de detritos y restos vegetales. Los detritos consistieron de pequeños gránulos de minerales y los vegetales incluyeron trozos de tallos, hojas y semillas indeterminadas.

El mayor IRI estuvo representado por los ordenes Díptera, Anfípoda y Arácnida, los que ha- 
cen un $50 \%$ de la dieta total de esta especie (Tabla I). Al considerar la dominancia es el ítem Tipulidae, que por número como por volumen, el que representa el principal ítem de consumo en $P$. thaul.

No existen diferencias significativas en la frecuencia de captura dentro de los seis ítemes más importantes de presa (Anyphanidae, Amphipoda, Araneidae, Muscidae, Drosophilidae y Tipulidae). Los resultados muestran que el consumo de estos ítems no difiere entre años $(\mathrm{G}=265.49 ; 5 \mathrm{gl} ; \mathrm{P}>$ $0.05)$.

Los valores de IRI para cada ítem, muestran que $P$. thaul consumió abundantes tipulidos durante ambos años, transformándose este ítem en el consumo principal (Tabla I). Sin embargo, durante el año 2000 los múscidos presentan la mayor frecuencia, en cambio los drosofílidos lo hacen en el año 2001. Para este anuro, tipulidos, anfípodos, múscidos, drosofílidos, anifánidos y araneidos son las categorías dominantes de presas de acuerdo a los valores de importancia (Tabla I), por lo que son utilizados para el análisis de selección por tamaño de presa.

Por otra parte se determinó que $P$. thaul pre- senta una baja amplitud dietaria, tanto en el volumen de las presas consumidas, como en el número de ítemes que componen su dieta (Fig. 1).

Relación tamaño de presa-depredador. Aún cuando $P$. thaul presenta un rango amplio de tamaños de presa (rango: 1.88 - 502.40), los resultados muestran un consumo más abundante de las presas con tamaños que fluctúan entre los $60-100 \mathrm{~mm}^{3}$, y una disminución en el consumo de aquellas presas que exceden los $100 \mathrm{~mm}^{3}$ (Fig. 2).

Los resultados muestran una ausencia de relación entre el tamaño de $P$. thaul y el volumen de las presas. De esta manera $P$. thaul (tamaño corporal, $\bar{X}=37,34 \mathrm{~mm} \pm 4,50 \mathrm{~mm}$ ) consumen un extenso rango de tamaños de presa $\left(\bar{X}=37,80 \mathrm{~mm}^{3}+\right.$ $\left.62,16 \mathrm{~mm}^{3}\right)$, sin que exista una relación entre el tamaño de éstas respecto al tamaño del depredador $\left(r^{2}=0,062, F=2,52, P=0.120\right)$.

El análisis de los seis taxa más importantes de acuerdo a los valores de IRI versus el tamaño corporal de los ejemplares, muestra una ausencia de correlación entre el tamaño de presa y el tamaño del depredador (Fig. 3).

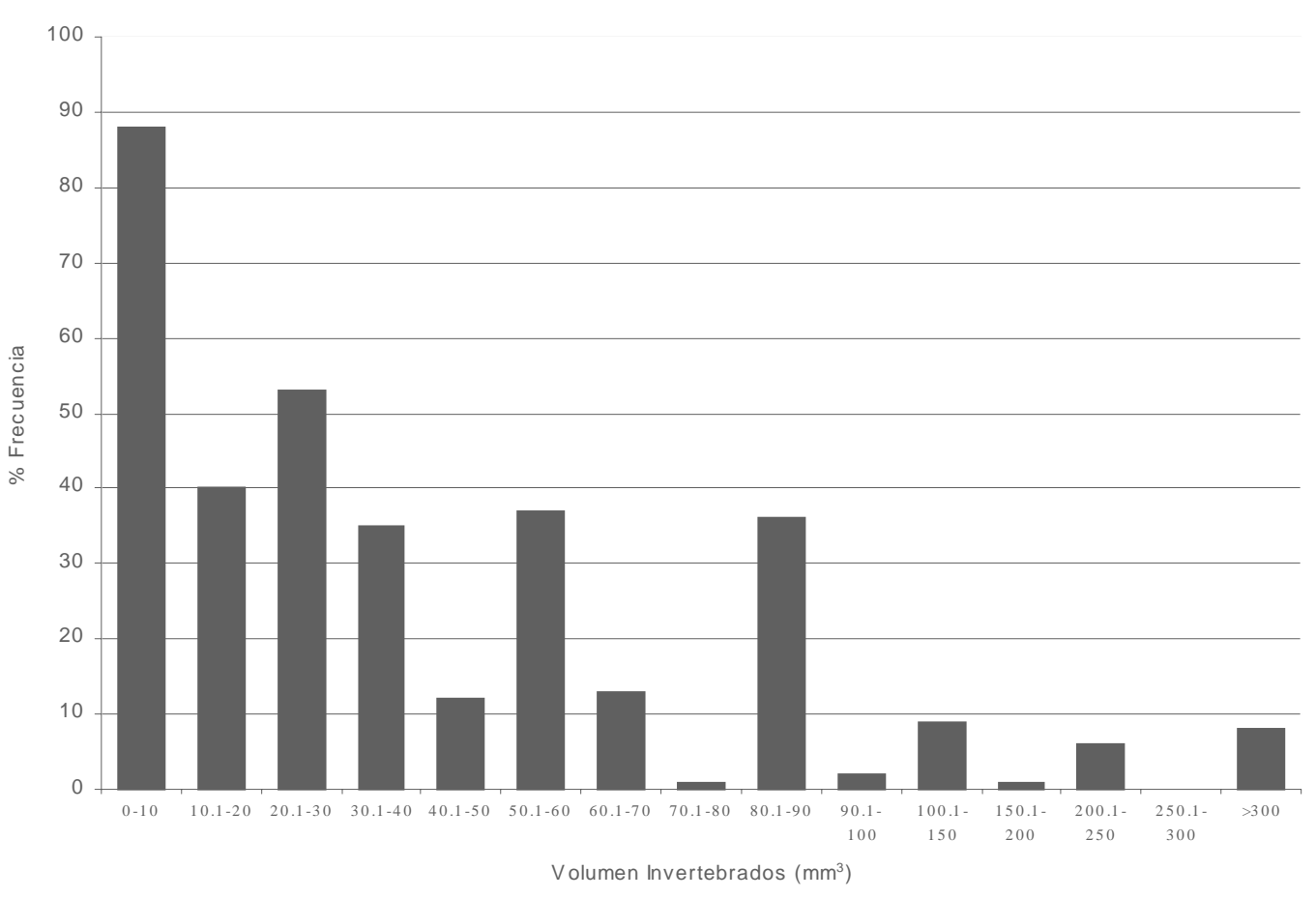

FIgURA. 2: Distribución de la frecuencia (\%) de las clases de volumen de presa en la dieta de Pleurodema thaul.

FIGURE. 2: Frequencys distribution (\%) of volume classes of preys of Pleurodema thaul. 

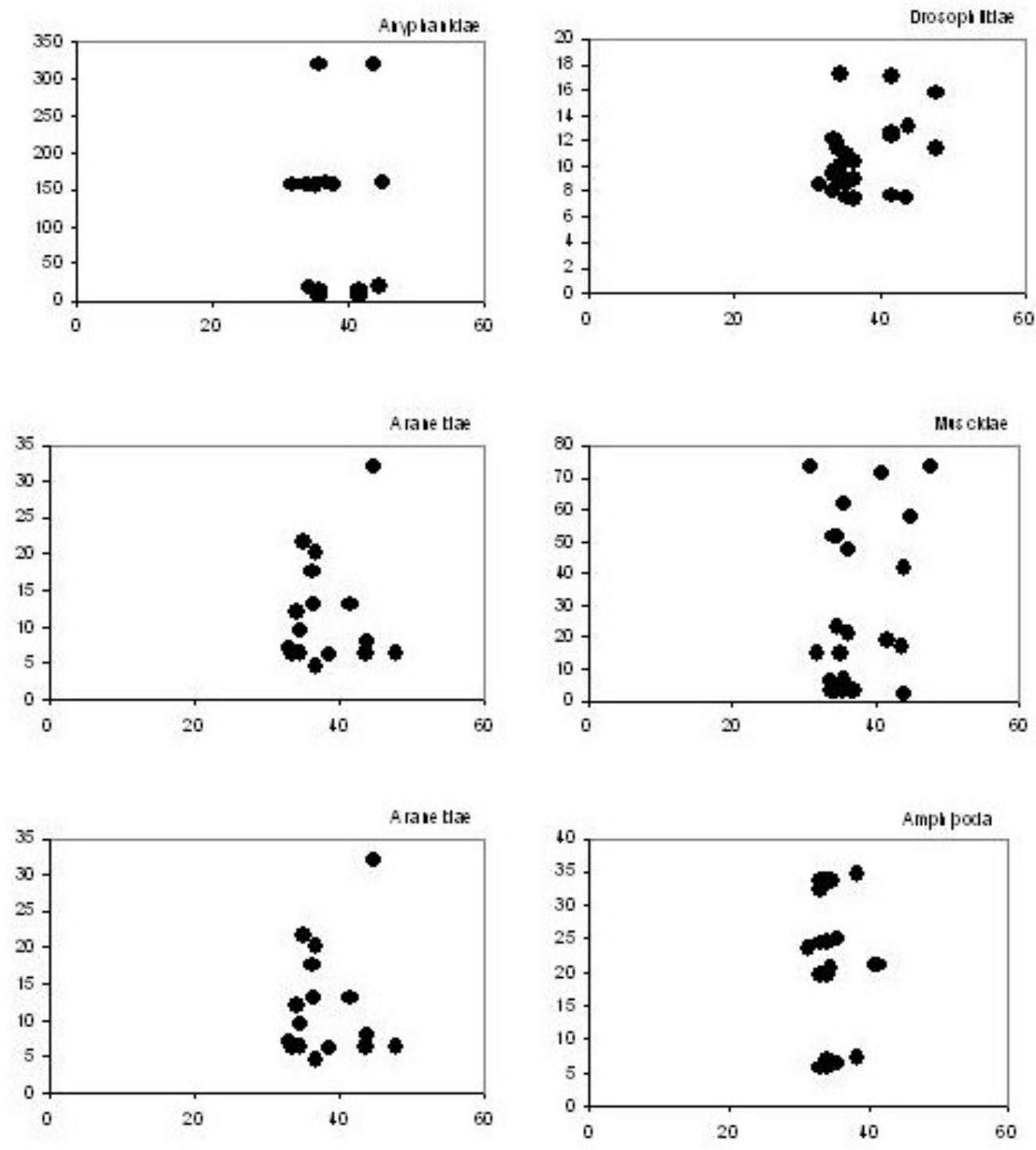

Tamaño Corporal (mm)

FiguRA. 3: Relaciones entre el tamaño corporal de los anuros y el volumen de los ítems de presa dentro de los seis más frecuentes órdenes de artrópodos. Anyphanidae $\mathrm{R}^{2}=0.006, \mathrm{P}=0.0491, \mathrm{n}=15$, Araneidae $\mathrm{R}^{2}=0.199, \mathrm{P}=0.185, \mathrm{n}$ $=16$, Drosophilidae $\mathrm{R}^{2}=0.178, \mathrm{P}=0.375, \mathrm{n}=27$; Muscidae $\mathrm{R}^{2}=0.152, \mathrm{P}=0.414, \mathrm{n}=31$; Tipulidae $\mathrm{R}^{2}=0.016, \mathrm{P}$ $=0.82, \mathrm{n}=121 ;$ Amphipoda $\mathrm{R}^{2}=0.02, \mathrm{P}=0.304, \mathrm{n}=26$.

FigURE. 3: Relationships between of body size and volume of ítems prey, in six more frequent order of arthropods. Anyphanidae $\mathrm{R}^{2}=0.491, \mathrm{P}=0.862, \mathrm{n}=15$, Araneidae $\mathrm{R}^{2}=0.739, \mathrm{P}=0.786, \mathrm{n}=16$, Drosophilidae $\mathrm{R}^{2}=0.178, \mathrm{P}$ $=0.375, \mathrm{n}=27$; Muscidae $\mathrm{R}^{2}=0.152, \mathrm{P}=0.414, \mathrm{n}=31$; Tipulidae $\mathrm{R}^{2}=0.016, \mathrm{P}=0.82, \mathrm{n}=121$; Amphipoda $\mathrm{R}^{2}=$ $0.02, \mathrm{P}=0.304, \mathrm{n}=26$. 


\section{DISCUSION}

Los resultados muestran que $P$. thaul posee un consumo mayoritario de dípteros, fundamentalmente tipulidos, en relación a los otros ítemes. Este consumo preferencial de los tipulidos se debe particularmente a que son presas grandes y nocturnas, lo cual coincide con los períodos máximos de forrajeo y actividad reproductiva de estos anuros (obs. personal).

En el análisis de los contenidos estomacales destaca la presencia de restos de vegetales. El consumo de vegetación es probablemente accidental, producido al momento de la captura de sus presas (Whitaker et al.1977; Stebbins \& Cohen 1995; Anderson et al. 1999). Sin embargo, no existe claridad respecto a la importancia del ítem vegetal dentro de la dieta de anuros. Algunos autores argumentan que los vegetales podrían ser consumidos activamente y utilizados como un medio de arrastre para ayudar en la eliminación de parásitos intestinales, y exoesqueletos de invertebrados. Otra posible utilización se refiere a una contribución a la nutrición, y/o como un recurso de agua adicional para prevenir desecación (Anderson et al. 1999).

Los escasos antecedentes dietarios en anfibios chilenos, reportan la presencia de abundante vegetación en una población de B. spinulosus (Núñez et al. 1982). Según estos autores, dichos anuros consumirían en forma selectiva microalgas, de manera de obtener nutrientes a partir de ellas. En nuestro estudio, existe un consumo de vegetales por parte de $P$. thaul $\left(X_{I R I}=0.035\right)$, y será necesario evaluar a futuro la probable función que cumple este ítem alimentario para la especie.

Para esta especie no existen antecedentes dietarios claros, sólo es posible encontrar las menciones al consumo como generalistas, realizadas por Cei $(1962,1980)$. Nuestros resultados en $P$. thaul muestran una tendencia a consumir tanto taxa de organismos terrestres como acuáticos (dípteros, anfípodos y arácnidos). La influencia de la disponibilidad de presas tanto en tipo como en cantidad ha sido reportada en diversas especies de anuros (Whittaker et al. 1977; Anderson et al. 1999).

Muchos anuros se alimentan de una variedad de organismos vivos sin que al parecer discriminen entre distintas presas. Sin embargo, se ha detectado que tanto los atributos de tamaño, mo- vimiento, palatabilidad, y valor nutritivo pueden afectar la selección que hacen los anuros del alimento (Anderson \& Mathis 1999; Anderson et al. 1999). Sin datos disponibles acerca de la oferta ambiental, no es posible referirse a una selección de los ítemes alimentarios determinados con mayor valor de importancia.

La depredación tamaño-selectiva es un rasgo común de las interacciones depredador-presa, para una variedad de ambientes acuáticos (Werner \& Hall 1974; Newman \& Waters 1984; Parker 1994). Basados en esta premisa, se ha determinado la ausencia de evidencias que demuestren que el número de presas, así como el tamaño de éstas, esté relacionado con la longitud corporal en $P$. thaul. Consecuentemente para esta especie de anuro, individuos grandes y pequeños consumen rangos de tamaño de presa similares.

\section{AGRADECIMIENTOS}

Los autores agradecen a Julio Becerra, José Castro, Daniel Pincheira-Donoso y Alejandra Rodríguez por su ayuda en la recolección de los datos en terreno. A las Dras. Eugenia Casanueva y Vivian Jerez, así como al biólogo Milenko Aguilera por su asistencia en la determinación de los invertebrados, y al profesor Hugo Moyano por facilitarnos el material óptico utilizado. Este trabajo forma parte de la tesis del primer autor para optar al grado de Doctor en Ciencias Biológicas (Departamento de Zoología, Universidad de Concepción). Este trabajo fue financiado parcialmente por el proyecto DIUC 92.3828, de la Universidad de Concepción y proyecto FONDECYT 89/1199.

\section{BIBLIOGRAFIA}

Anderson, A., Haukos, D. \& J. Anderson. 1999. Diet composition of three anurans from the Playa wetlands of northwest Texas. Copeia 1999: 515520.

Anderson, MT \& A. Mathis. 1999. Diets of two sympatric Neotropical Salamanders, Bolitoglossa mexicana and B. rufescens, with notes on reproduction for $B$. rufescens. J. Herpetol. 33: 601-607.

Artigas, J. 1994a. Entomología Económica. Insecta de Interés Agrícola, Forestal, Médico y Veterina- 
rio (Nativos, Introducidos y Susceptibles de ser Introducidos). Vol 1. Ediciones Universidad de Concepción: 1.126 pp.

Artigas, J. 1994b. Entomología Económica. Insecta de Interés Agrícola, Forestal, Médico y Veterinario (Nativos, Introducidos y Susceptibles de ser Introducidos). Vol 2. Ediciones Universidad de Concepción: 943 pp.

Boice, R. \& R.C. Williams. 1971. Competitive feeding behavior of Rana pipiens and Rana clamitans. Anim. Behav. 19: 548-551.

CeI, J.M. 1962. Batracios de Chile. 128 pp. Lam. y Figs. Ed. Universidad de Chile.

CEI, J.M. 1980 Amphibians of Argentina. Monitore Zoologico Italiano (N.S.) Monografía 2,609 pp.

Formas, R. 1995. Anfibios. Pp: 314-325. En: Diversidad biológica de Chile. Edit. Simonetti, J.A., Arroyo, M.TK., Spotorno, A. \& Lozada, E.. CONICYT: $364 \mathrm{pp}$.

JoNES, K. 1982. Prey patterns and trophic niche overlap in four species of Caribbean frogs: $49-55 \mathrm{pp}$. In: Herpetological Communities. United States Department of the interior fish and wildlife service. Wildlife Research Report 13: 239.

Livaitis, J.A., K. Titus \& E.M. Anderson. 1994. Measuring vertebrates use of terrestrial habitats and foods. Pp: 254-274. En: Research and management techniques for wildlife and habitats. T.A. Bookhout (ed.) 5th ed. Wildlife Society, Bethesda, MD.

MaIorana, V.C. 1978. Behavior of an unobservable species: diet selection by a salamander. Copeia 1978: 664672.

Murphy, Chr. 1992. The mating of the barking treefrog (Hyla gratiosa). Ph D. Dissertation, Cornell University.

Newman, R.M. \& T.F. Waters. 1984. Size-selective predation on Gammarus pseudolimnius by trout and sculpins. Ecology 65: 1535-1545.

NúÑEZ, H., M.A. Labra \& J. NúÑ̃z. 1982. Hábitos alimentarios de dos poblaciones andinas de Bufo spinulosus Wiegmann, 1985 (Anura: Bufonidae). Bol. Mus. Nac. Hist. Nat. Santiago 39: 81-91.

PARKER, M. 1994. Feeding ecology of stream-Dwelling Pacific Giant Salamander Larvae (Dicamptodon tenebrosus). Copeia 1994: 705-718.

PeÑA, L. 1988. Introducción a los insectos de Chile. Editorial Universitaria: $254 \mathrm{pp}$.

Petranka, J.W. 1984. Ontogeny of the diet and feeding behavior of Eurycea bislineata larvae. J. Herpetol. 18: 48-55.

Pianka, E.R. 1974. Niche overlap and diffuse competition. Proc. Nat. Acad. Sci. U.S.A. 71: 2141-2145.

Pincheira-Donoso, D. 2002a. Nota sobre la alimentación de Pleurodema bufonina Bell 1843 (Anura - Leptodactylidae). Gayana 66: 77-80.

Pincheira-Donoso, D. 2002b. Dieta de Batrachyla taeniata (Girard, 1854) en poblaciones de Concepción, Chile (Anura: Leptodactylidae). Not. Mens. Mus. Nac. Hist. Nat. Santiago: 3-7.

Powell, R., J.S. Parmelee, JR., M.A. Rice \& D.D. Smith. 1990. Ecological observations of Hemidactylus brooki haitianus Meerwath (Sauria: Gekkonidae) from Hispanoila. Carib. J. Sci. 26: 67-70.

Simpson, E.H. 1949. Measurement of diversity. Nature 163: 688.

Stebbins, R.C. \& N. W. Cohen. 1995. A Natural History of Amphibians. Princeton Univ. Press, Princeton, N.J.

Valencia, J., A. Veloso \& M. Sallaberry. 1982. Nicho trófico de las especies de herpetozoos del transecto Arica-Chungará. Pp: 269-291 En: El ambiente natural y las poblaciones humanas de los Andes del norte grande de Chile (Arica, Lat. 18 28'S). Veloso, A. \& E. Bustos-Obregón (eds.) Volumen I, Proyecto MAB-6, UNEP-UNESCO 1105-77-01, ROSTLAC, Montevideo.

WeLLs, K.D. 1978. Territoriality in the green frog (Rana clamitans): Vocalizations and agonistic behavior. Anim. Behav. 26: 1051-1063.

Werner, E.E. \& D.J. HaLl. 1974. Optimal foraging and the size selection of prey by the bluegill sunfish (Lepomis macrochirus). Ecology 55: 1.0421.052.

Whittaker, JR. J.O., D. Rubin \& J.R. Munsee. 1977. Observations on food habits of four species of spadefoot toads, genus Scaphiopus. Herpetologica 33: 468-475. 